\title{
Cultura Organizacional, Nível de Parceria da Controladoria e Sistemas de Avaliação de Desempenho
}

\author{
Marília Janaina da Silva Bassi \\ https://orcid.org/0000-0002-4584-1351 \\ Paschoal Tadeu Russo \\ https://orcid.org/0000-0002-7293-4385
}

José Carlos Tiomatsu Oyadomari https://orcid.org/0000-0003-3059-3102

Maria Thereza Pompa Antunes https://orcid.org/0000-0002-9501-2789

\section{Resumo}

Objetivo: Compreender as associações existentes entre as diferentes intensidades de tipificações de cultura organizacional (CO), níveis de parceria exercidos pelo setor de controladoria (NPC) e a amplitude dos sistemas de avaliação de desempenho organizacional (ASADO).

Método: Estudo descritivo, com abordagem quantitativa, por meio de uma survey com 89 respondentes, especialmente controllers, cujas percepções foram avaliadas de acordo com as três variáveis mencionadas, mediante análise multivariada (ANACOR e HOMALS).

Resultados: Os resultados revelam que organizações com culturas organizacionais mais fortes estão diretamente associadas a setores de controladoria mais participativos (níveis mais elevados de parceria da controladoria), os quais utilizam sistemas de avaliação de desempenho mais amplos. Mostram, ainda, ausência de associação direta entre cultura organizacional e amplitude de sistemas de avaliação de desempenho.

Contribuições: Para a academia, evidenciar a compreensão da associação direta entre NPC e ASADO e a impossibilidade de mostrar associação direta entre CO e ASADO. Com isso, o estudo transcende as usuais abordagens explicativas da Teoria da Contingência. Para a prática profissional, sobretudo para os responsáveis pela área de controladoria, oferece uma visão clara da associação de culturas organizacionais fortes com níveis superiores de parceria da área, e desta com a maior amplitude dos sistemas de avaliação desenvolvidos pela área.

Palavras-chave: Cultura Organizacional, Controladoria como parceira de negócios, Sistema de Avaliação de Desempenho. 


\section{Introdução}

Os relacionamentos contingentes da variável Cultura Organizacional (Schein, 1984; Smircich, 1983) com as variáveis Estrutura Organizacional e Desempenho (Carmona, Silva, \& Gomes, 2018; Gordon \& DiTomaso, 1992; Kotrba et al., 2012; Parente et al., 2018; Smircich, 1983; Zheng, Yang, \& McLean, 2010) vêm sendo estudados há algumas décadas (O’Reilly et al., 2014). Adicionalmente, algumas pesquisas evidenciaram a associação entre a força da cultura (que também pode ser denominada intensidade da cultura e medida por meio da consistência, ou centralidade, das respostas obtidas nas pesquisas, relativamente a padrões ou valores culturais específicos do contexto organizacional estudado) e sua influência no desempenho (Lee \& Yu, 2004; Gordon \& DiTomaso, 1992).

Trazendo essa reflexão para o campo da Contabilidade Gerencial, pode-se considerar que a controladoria, enquanto área administrativa (Catelli, 2001; Melo \& Paulo, 2000), é parte da estrutura organizacional, e, de forma contingente, inclusive por meio da adequação dos serviços por ela prestados, se ajusta às necessidades da organização, aos demais fatores contingenciais, dentre os quais a Cultura Organizacional, e assim participa ativamente da elaboração e uso de diversos artefatos que visam contribuir com os gestores em seus processos de decisão (Tarifa et al., 2011; Henri, 2006).

O processo de evolução da controladoria e do controller tem sido tema de diversas pesquisas, sobretudo as que avaliam as atividades a eles associadas (Beuren, Fachini \& Nascimento, 2010; Borinelli, 2006; Catelli, 2001; Tarifa et al., 2011); entretanto, alguns autores (Goretzki, Strauss \& Weber, 2013; Järvenpää, 2007; Weber, 2011) têm proposto um novo enfoque de pesquisa, ao avaliar a controladoria como "Parceira de Negócios" (business partner), à medida que ela e o próprio controller passam a desenvolver tarefas mais proativas perante as áreas de negócio (Weber, 2011). De certa forma, a literatura está alinhada com o que acontece na prática, pois o termo Business Partner está incorporado à realidade organizacional, como se vê na literatura de negócios e também nas empresas, como no caso da BASF, que adota esse termo (Arenales, 2016).

Também existem críticas em relação à falta de clareza e objetividade dos sistemas de avaliação de desempenho, em termos de sua amplitude e propósito, quanto ao que é avaliado (Neely, Bourne, \& Kennerly, 2000; Neely, Gregory, \& Platts, 1995), pois é a partir deles que se define o que, como e quando deverá ser mensurado (Merchant \& Van der Stede, 2007), e usado como base para a avaliação do desempenho. Em suma, Sistemas de Avaliação de Desempenho podem ser compreendidos como artefatos ou construções que oferecem respostas às necessidades informacionais dos gestores e que também estão sujeitos a ajustes decorrentes de demandas dos diferentes elementos dos contextos interno e externo das organizações.

De outro lado, a Cultura Organizacional pode ser compreendida como um recurso estratégico relevante (Parente et al., 2018; Santos,1998), sendo que determinadas formas de Cultura Organizacional, sobretudo as que mostram tipificações mais fortes, poderão estar associadas a ajustes nas demais estruturas existentes (Santos et al., 2014; Smircich, 1983; Lee \& Yu, 2004; Gordon \& DiTomaso, 1992). Este é o caso da área de controladoria, que pode estar associada a tarefas e processos realizados de forma distinta, resultando em diferentes desempenhos (Tarifa \& Almeida, 2018; Bonisenha \& d’Angelo, 2018; Parente et al., 2018; Santos,1998).

Diversos esforços vêm sendo desenvolvidos no sentido de evidenciar as possibilidades de contribuição da área de controladoria e do papel do controller nas organizações (Järvenpää, 2007; Weber, 2011; Goretzki et al., 2013; Arenales, 2016). Entretanto, as dificuldades encontradas pelos profissionais que tentam implementar a controladoria como Parceira de Negócios ainda são grandes, e a compreensão acerca da associação entre fatores culturais e o desenvolvimento de sistemas de avaliação de desempenho pode contribuir para a evolução na forma como a controladoria se desenvolve nessas organizações, caracterizada em termos de Nível de Parceria. Assim, este estudo tem por objetivo ampliar a compreensão sobre as associações entre as diferentes intensidades dos tipos de Cultura Organizacional, níveis de parceria na controladoria e a amplitude dos sistemas avaliação de desempenho. 
Com base nos argumentos apresentados, e buscando estender o alcance do que em estudos anteriores foi pesquisado de forma isolada, define-se a questão que norteia esta pesquisa: "Quais são as associações existentes entre as diferentes forças dos tipos de cultura organizacional, o nível de parceria exercido pelo setor de controladoria, e a amplitude dos sistemas de avaliação de desempenho organizacional?"

A fim de investigar empiricamente esta questão, foi realizado um levantamento (survey) com 89 profissionais que trabalham em empresas não financeiras, no Brasil, e nas quais a controladoria é um órgão administrativo.

A pesquisa também contribui para a literatura sobre o tema, buscando evidenciar as associações entre artefatos, estruturas e tipificações culturais (Schein, 1984), além de mostrar como as características das variáveis Cultura Organizacional, Níveis de Parceria da Controladoria e Amplitude dos Sistemas de Avaliação de Desempenho são identificadas nos diversos contextos organizacionais, com foco na melhoria do desempenho da área de controladoria (Oyadomari et al., 2014).

\section{Revisão da Literatura}

Nos itens seguintes, apresenta-se a revisão da literatura que embasou a formulação das hipóteses desta pesquisa, acerca das associações entre as variáveis Cultura Organizacional, Níveis de Parceria da Controladoria e Amplitude dos Sistemas de Avaliação de Desempenho.

\subsection{Características da Cultura Organizacional e da Controladoria como Business Partner}

Schein (1984) conceitua a Cultura Organizacional como um padrão dinâmico de premissas básicas definidas por um grupo, como resposta a problemas e que são ensinadas a novos membros, caracterizando, assim, "a maneira correta de perceber, pensar e sentir em relação a esses problemas (Schein, 1984, p. 3). Ela também pode ser compreendida como "a cola social" ou normativa que promove a coesão organizacional (Schein, 1984, p. 14; Smircich, 1983), funcionando como um mecanismo de controle, ainda que informal, aprovando ou proibindo comportamentos; dá significado, direção, mobilização e motivação para os membros da organização; manifesta-se por meio de práticas, comportamentos e artefatos compartilhados entre os membros de uma empresa (Pothukuchi et al., 2002; Cao et al., 2015). A literatura mostra que as áreas e unidades de negócio de uma empresa tendem a reagir melhor quando as práticas de gestão adotadas são compatíveis com o modelo de cultura organizacional vigente (Pothukuchi et al., 2002).

Pesquisas têm mostrado que culturas percebidas como mais fortes, ou seja, nas quais existe maior nível de consenso (Ho, Wu \& Wu, 2014), ou que possuem valores e regras mais claramente definidos, mais consistentes e estáveis, independentemente de seu valor substantivo, estão associadas à maior adaptabilidade e melhor desempenho (Gordon \& DiTomaso, 1992; Hansen \& Wernerfelt, 1989; Lee \& Yu, 2004). Apesar de haver evidências de que a Cultura Organizacional influencia a Estrutura Organizacional (Smircich, 1983; Zheng \& McLean 2010), não se identificou se as diferentes percepções da força da cultura têm implicações diretas em tal associação, como já ocorreu em relação ao desempenho (Lee \& Yu, 2004; Gordon \& DiTomaso, 1992).

Cameron e Quinn (2005) desenvolveram um modelo denominado Organizational Culture Assessment Instrument (OCAI), que possibilita identificar as características culturais de uma determinada organização, inclusive a existência de padrões predominantes, ou seja, mais fortes. Inúmeras pesquisas têm se valido do modelo desenvolvido por Cameron e Quinn (2005) (Barreto et al., 2013; Dubey et al., 2019; Hartnell, Ou, \& Kinicki, 2011; Hock-Doepgen et al., 2021; Naranjo-Valencia, Jiménez-Jiménez, \& Sanz-Valle, 2011; Schein, 2010; Sony, Antony \& Douglas, 2020; Tian et al., 2018). 
O Modelo OCAI apresenta quatro grupos de valores culturais, com características específicas, que podem ser observados nas organizações: (i) Cultura Clã: apresenta similaridade com empresas familiares, tendo como características a valorização da participação dos membros da empresa no processo decisório, no trabalho em equipe e no desenvolvimento de recursos humanos; (ii) Cultura Inovativa: voltada para um processo de inovação e motivada por acontecimentos externos à empresa, tendo por características a liderança criativa e motivadora, a diversificação das operações internas e a capacidade de flexibilidade e reestruturação; (iii) Cultura Hierárquica: os objetivos e as ações a serem executados pelas áreas e unidades de negócio organizacionais são definidos pela alta cúpula administrativa, tendo como características fatores motivacionais burocráticos e conservadores; e (iv) Cultura de Mercado: não se refere aos aspectos tradicionais de um mercado consumidor, e, sim, a um tipo de mercado próprio, com base nas influências externas e nas atividades específicas da empresa, nos resultados, nos clientes e nos demais fatores externos que contribuem para o desenvolvimento da companhia, tendo como características o foco na competitividade e na produtividade.

Pesquisas também mostram que a controladoria tem obtido melhor desempenho à medida que passa a ter uma postura orientada para a estratégia organizacional, atuando com um alto nível de envolvimento e comprometimento com o negócio global da empresa, de modo cooperativo com as demais áreas e unidades de negócio, o que tem sido atribuído a uma postura de parceira de negócios (Järvenpää, 2007; Weber, 2011, Goretzki et al., 2013). Isso porque ao atuar em maiores níveis de parceria, a controladoria pode contribuir, de forma mais ativa e direta, para a gestão estratégica organizacional (Weber, 2011; Rieg, 2018).

A Figura 1 apresenta o quadro conceitual elaborado por Weber (2011, p. 42), em que o autor buscou evidenciar os diferentes níveis de atuação do setor de controladoria, as ligações estreitas existentes entre eles e, ainda, as diversas atividades que são realizadas nos diferentes níveis. Ela possibilita caracterizar, dentre quatro estágios apresentados, o estágio de desenvolvimento e/ou de maturidade de uma dada controladoria, em função das atividades desempenhadas. As faixas que podem ser identificadas na Figura 1 são denominadas neste trabalho 'estágios de maturidade da área de controladoria' e são dispostas da menor para a de maior maturidade, de baixo para cima. No quarto e último estágio, de forma proativa, a controladoria fornece informações, contribui para a elaboração de planos, desafiando e estimulando a gestão por meio de um novo olhar sobre o negócio e sobre o contexto em que ele está inserido. Nesta última fase, pode-se considerar que a controladoria assume o comportamento de parceira de negócios (Weber, 2011).

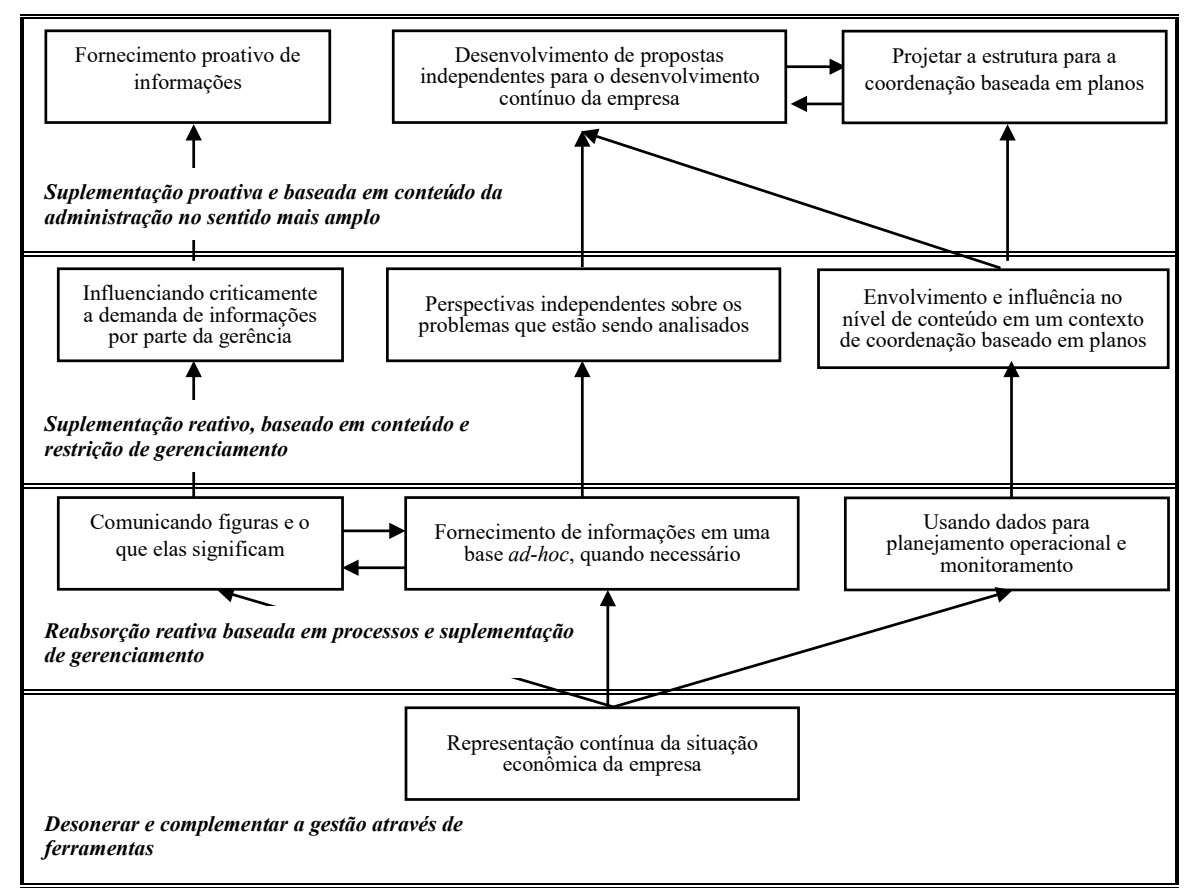

Figura 1. quadro conceitual de evolução da controladoria

Fonte: Weber (2011 p. 42). 
O processo evolutivo da controladoria, conforme descrito, ocorre por meio da progressividade de suas atividades, que leva em conta a eliminação de 'gargalos' de racionalidade no seu desenvolvimento, em função do aumento da maturidade já alcançada pelo setor (Weber, 2011 p. 27). Para tanto, o quadro conceitual apresenta, para cada um dos níveis, a gama de tarefas consideradas como sendo de responsabilidade do setor, considerando que as atividades dos estágios anteriores não são abandonadas com o processo evolutivo, mas adicionadas às novas tarefas decorrentes do processo (Weber, 2011).

De acordo com o nível (ou estágio) alcançado pelo setor, este atuará de forma mais ou menos ativa, como um parceiro de negócios das demais áreas e unidades de negócio da empresa, ou seja, poderá ser mais ou menos percebido como um business partner. Com base nos elementos apresentados, sugere-se a primeira hipótese:

Hipótese 1: Tipificações culturais mais fortes estão diretamente associadas a níveis mais elevados de parceria de negócios da controladoria.

\subsection{Características da Cultura Organizacional e dos Sistemas de Avaliação de Desempenho}

Bititci et al. (2012, pp. 24-25) observam que o modelo de cultura organizacional e a natureza do trabalho conduzido em uma organização influenciam a forma como seu desempenho é medido e gerenciado. A literatura relacionada aos Sistemas de Avaliação de Desempenho mostra a influência da cultura organizacional, e, em decorrência, dos estilos de gestão, para o sucesso da implementação de tais sistemas (Bititci et al., 2006); sobretudo aquelas que buscam não punir as pessoas, mas incentivam a discussão e análise, têm melhores resultados (Franco \& Bourne, 2003).

"A mensuração de desempenho é um tópico frequentemente discutido, mas raramente definido. Literalmente, é o processo de quantificar a ação, onde a medição é o processo de quantificação e a ação leva ao desempenho" (Neely, Gregory \& Platts, 1995, p. 80). Um dos fatores que levam a isso é que autores individuais tendem a se concentrar em diferentes aspectos do desenho do sistema de medição de desempenho. Em consequência das diferentes visões e objetivos, comumente os sistemas de avaliação de desempenho visam atender a necessidades específicas: alguns buscam avaliar desempenhos em pequenas e médias empresas (Garengo, Biazzo, \& Bititci, 2005), outros estão voltados à mensuração e à comunicação do desempenho esperado pelos diversos stakeholders (Silva, Nuzum, \& Schaltegger, 2019). Atualmente, enfatiza-se a importância de sistemas que auxiliem a mensuração do desempenho à luz da sustentabilidade dos negócios e organizações (Cagno et al., 2019; Silva et al., 2019). 
Neely et al. (2000), ao consolidarem uma série de proposições a partir de Globerson (1985) e Maskell (1991), desenvolveram um quadro conceitual para identificar o conjunto de atributos desejáveis a um sistema de avaliação de desempenho (Tabela 1). Ele mostra a amplitude de tais sistemas por meio de duas dimensões: 1) características desejáveis para o desenvolvimento de um sistema de avaliação de desempenho (desenho); e 2) características desejáveis para o seu uso (avaliação do processo). Este foi o quadro conceitual usado como base para o nosso instrumento de pesquisa.

Tabela 1

\section{Características de um Sistema de Avaliação de Desempenho}

\begin{tabular}{|c|c|}
\hline $\begin{array}{l}\text { Características desejáveis no desenvolvimento de um } \\
\text { sistema de medição de desempenho }\end{array}$ & $\begin{array}{l}\text { Características desejáveis para uso do sistema de } \\
\text { medição de desempenho (avaliação do processo) }\end{array}$ \\
\hline $\begin{array}{l}\text { O processo deve ser revisto facilmente - as medidas } \\
\text { devem mudar conforme mudem as circunstâncias }\end{array}$ & $\begin{array}{l}\text { As medidas de desempenho devem permitir/facilitar o } \\
\text { benchmarking }\end{array}$ \\
\hline $\begin{array}{l}\text { O objetivo de cada medida de desempenho deve ser } \\
\text { evidenciado }\end{array}$ & $\begin{array}{l}\text { Critérios de desempenho objetivos são preferíveis aos } \\
\text { subjetivos }\end{array}$ \\
\hline $\begin{array}{l}\text { A coleta de dados e métodos de cálculo do nível de } \\
\text { desempenho devem ser claros }\end{array}$ & $\begin{array}{l}\text { As medidas de desempenho baseadas na razão são } \\
\text { preferíveis a números absolutos }\end{array}$ \\
\hline $\begin{array}{l}\text { Todos (clientes, funcionários e gerente) devem estar } \\
\text { envolvidos na seleção das medidas }\end{array}$ & $\begin{array}{l}\text { Os critérios de desempenho devem estar sob o controle } \\
\text { direto da unidade organizacional avaliada }\end{array}$ \\
\hline $\begin{array}{l}\text { As medidas de desempenho selecionadas devem levar em } \\
\text { conta a organização }\end{array}$ & $\begin{array}{l}\text { As medidas de desempenho devem estimular a melhoria } \\
\text { contínua, em vez de apenas monitorar }\end{array}$ \\
\hline \multirow[t]{3}{*}{$\begin{array}{l}\text { As medidas de desempenho devem ser derivadas da } \\
\text { estratégia da empresa }\end{array}$} & $\begin{array}{l}\text { As medidas de desempenho devem ser simples e fáceis } \\
\text { de usar }\end{array}$ \\
\hline & $\begin{array}{l}\text { As medidas de desempenho devem fornecer feedback } \\
\text { rápido }\end{array}$ \\
\hline & Medidas não financeiras devem ser adotadas \\
\hline
\end{tabular}

Fonte: Neely et al. (2000, p. 1131), configurada com base em Globerson (1985) e Maskell (1991).

Neste estudo, define-se Amplitude do Sistema de Avaliação de Desempenho como um constructo capaz de operacionalizar a combinação de características presentes no desenho e uso desse sistema, com base no quadro conceitual de Neely et al. (2000).

Por fim, os Sistemas de Avaliação de Desempenho são artefatos decorrentes da cultura da organização (Schein, 1984) e resultam dos diferentes elementos do seu contexto interno, entre os quais a força das diferentes tipificações de Cultura Organizacional, que é um recurso estratégico relevante (Parente et al., 2018; Santos,1998). Pode-se, então, considerar que a amplitude com que tais sistemas são desenvolvidos e utilizados resulta da força da cultura (medida pela consistência das respostas obtidas nas pesquisas, relativamente à adaptabilidade e estabilidade organizacionais).

Com base nos elementos apresentados, sugere-se a segunda hipótese:

Hipótese 2: Tipificações culturais mais fortes estão diretamente associadas a maiores amplitudes dos sistemas de avaliação de desempenho. 
Por outro lado, pesquisas têm mostrado associações entre diferentes tipificações culturais e diferentes conjuntos de práticas de Contabilidade Gerencial (Tarifa \& Almeida, 2018), ou mesmo em processos relacionados à gestão de pessoas (Santos et al., 2014). Além disso, os sistemas de avaliação de desempenho, ao longo de seu processo de adoção e uso, passam por adequações decorrentes do amadurecimento da cultura e das estruturas a eles associados (Bititci et al., 2006). Pode-se depreender que, nas controladorias com níveis mais elevados de parceria, existem elementos conceituais, conhecimentos e sistemas operantes, que decorrem de níveis mais elevados de agregação, integração e escopo e que garantem a elaboração de relatórios tempestivos (Frezatti, Relvas \& Junqueira, 2010), possibilitando desenvolver Sistemas de Avaliação de Desempenho mais amplos ou mais sofisticados. Isto possibilitou sugerir a terceira hipótese:

Hipótese 3: Níveis mais elevados de parceria de negócios da controladoria estão diretamente associados a maiores amplitudes dos sistemas de avaliação de desempenho.

\section{Procedimentos Metodológicos}

Este estudo se caracteriza como uma pesquisa descritiva e quantitativa, com corte transversal, do tipo survey. Para a coleta dos dados, a qualidade, confiabilidade e aplicabilidade do questionário desenvolvido foram testadas por meio do coeficiente Alfa de Cronbach (Hora, Monteiro \& Arica, 2010), paralelamente à realização de um pré-teste aplicado a oito profissionais da área de controladoria, escolhidos por tipicidade, e que não fizeram parte da amostra final. Com isso, foi possível eliminar pontos que poderiam gerar dúvidas, além de mensurar o tempo médio de resposta.

O levantamento foi realizado por meio de uma web survey (Miranda, Riccio \& Miranda, 2012, p. 119), o que possibilitou alcançar um maior número de respondentes, além de reduzir os tradicionais custos de pesquisa (Dillman, 2000). A principal fonte utilizada para a identificação de participantes da pesquisa foi a rede social profissional LinkedIn de um dos pesquisadores.

Os questionários foram coletados no período compreendido entre setembro e outubro de 2018, sendo obtidos 119 retornos, de um total de 336 contatos da rede da pesquisadora, que tinham o perfil funcional alinhado aos objetivos da pesquisa, ou seja, profissionais que atuavam no processo de gestão e utilizavam sistemas para a avaliação de desempenho desenvolvidos pela controladoria, em suas organizações. Isto representou 35,42\% dos questionários enviados. Após excluídas as respostas que não possibilitavam assegurar o envolvimento direto com as três dimensões pesquisadas - cultura organizacional, controladoria e sistemas de avaliação de desempenho -, a amostra final foi composta por 89 profissionais, sendo 48 controllers, 26 gerentes de controladoria, quatro diretores, dois Chief Operating Officers (COO), e nove com outras funções. Esses profissionais atuavam majoritariamente em empresas de médio e grande porte ( 34 grandes e 45 médias), e apenas 10 em pequenas empresas.

Os resultados foram obtidos pela utilização de análise multivariada de dados (Figueira, 2004a,b; Fávero et al., 2009), de acordo com a Análise de Correspondência (ANACOR), modelo aplicado ao estudo da relação entre duas variáveis qualitativas.

As variáveis latentes "amplitude do sistema de avaliação de desempenho" e "nível de parceria exercido pelo setor de controladoria", foram mensuradas por meio de variáveis categóricas, enquanto a variável "cultura organizacional" foi medida pelo constructo OCAI. 
As categorias apresentadas na Tabela 2 são decorrência direta da utilização do instrumento OCAI e dos testes estatísticos aplicados.

Tabela 2

Categorização do Tipo de Cultura Organizacional (Cat_Cult)

\begin{tabular}{ccc}
\hline Sigla & Tipificação Cultural & Quantidade \\
\hline Cla & Dominância de cultura clã & 30 \\
\hline Ino & Dominância de cultura inovativa & 7 \\
\hline Hie & Dominância de cultura hierárquica & 32 \\
\hline Mer & Dominância de cultura de mercado & 16 \\
\hline Cla_Hi & $\begin{array}{c}\text { Modelo cultural misto, com proporções equivalentes de aspectos culturais } \\
\text { predominantemente associados à cultura clã e à cultura hierárquica }\end{array}$ & 1 \\
\hline Ino_Hi & $\begin{array}{l}\text { Modelo cultural misto, com proporções equivalentes de aspectos culturais } \\
\text { predominantemente associados à cultura inovativa e à cultura hierárquica }\end{array}$ & 3 \\
\hline
\end{tabular}

Com base nos resultados obtidos, referentes à parte 2 do questionário (Tabela 2), o modelo cultural hierárquico (Hie) foi o predominante entre as empresas analisadas, seguido do modelo cultural clã (Cla), o que corrobora parcialmente os achados de Tarifa e Almeida (2018), que encontraram em sua amostra predominância de empresas com tipificação cultural grupal (clã), seguida da hierárquica.

Para as categorias advindas dos instrumentos de pesquisa Níveis de Parceria da Controladoria (Tabela 3) e Amplitude dos Sistemas de Avaliação de Desempenho (Tabela 4), buscou-se estabelecer categorias que expressassem relacionamento direto com as caraterísticas pesquisadas (vide as descrições das categorias nas Tabelas 3 e 4); com base nessa estrutura, as pontuações obtidas foram classificadas em grupos: seis para o primeiro instrumento e cinco para o segundo.

Tabela 3

Categorização do Nível de Parceria do Setor de Controladoria (Cat_Contr)

\begin{tabular}{|c|c|c|}
\hline Sigla & Descrição dos Níveis de Parceria da Controladoria & Quantidade \\
\hline BP_F & $\begin{array}{c}\text { "Fraco": evidencia que o setor de controladoria da empresa está limitado ao } \\
\text { fornecimento reativo de dados e informações }\end{array}$ & 6 \\
\hline BP_B & $\begin{array}{l}\text { "Baixo": evidencia que o setor de controladoria da empresa, além das atividades relativas } \\
\text { ao nível anterior, também transmite as informações e as utiliza para fins de planejamento }\end{array}$ & 2 \\
\hline BP_M & $\begin{array}{l}\text { "Médio1": demonstra que o setor de controladoria da empresa, além das atividades } \\
\text { relativas aos estágios anteriores, também fornece perspectivas independentes sobre as } \\
\text { informações, e atua na coordenação dos planos de ação da empresa }\end{array}$ & 2 \\
\hline BP_R & $\begin{array}{l}\text { "Médio2": demonstra que o setor de controladoria fornece propostas independentes } \\
\text { para as necessidades identificadas pela área, desenvolve e coordena os ritos, mas não } \\
\text { realiza de forma satisfatória as atividades dos níveis anteriores }\end{array}$ & 13 \\
\hline BP_A & $\begin{array}{l}\text { "Alto": demonstra que o setor de controladoria atua de forma satisfatória no } \\
\text { fornecimento de propostas independentes para as necessidades identificadas pela área, } \\
\text { desenvolve e coordena os ritos como protagonista, e atende de forma satisfatória às } \\
\text { atividades que lhe cabem nos demais estágios de atuação }\end{array}$ & 31 \\
\hline BP_S & $\begin{array}{c}\text { "Avançado": demonstra que o setor de controladoria desenvolve, com um alto nível de } \\
\text { atuação, o fornecimento de propostas independentes para as necessidades identificadas } \\
\text { pela área, desenvolve e coordena os ritos como protagonista, e atende de forma } \\
\text { satisfatória às atividades que lhe cabem nos demais estágios de atuação, podendo, } \\
\text { assim, ser considerado um setor parceiro de negócios }\end{array}$ & 35 \\
\hline
\end{tabular}


Quanto à parte 3 do questionário (Tabela 3), pode-se identificar a predominância de empresas (74\%) com controladorias que podem ser caracterizadas por um elevado nível de parceria (BP_A = 31 e BP_S = 35). Não foi possível confrontar tais achados com a literatura, uma vez que ainda são poucos os estudos que categorizam as funções da controladoria como parceira de negócios (Arenales, 2016; Silva, 2017; Lunkes, Schnorrenberger \& Alexadre, 2016; Järvenpää, 2007; Weber, 2011), e seus resultados não possibilitam comparações, uma vez que são, em geral, estudos de caso.

Quanto à parte 4 do questionário (Tabela 4), identificou-se a predominância (66\%) de percepção da existência de sistemas de avaliação de desempenho com maior amplitude (SD_A = 49 e SD_S=89) das empresas analisadas, apesar de, na mesma tabela, também se observar que $22 \%$ dos respondentes categorizaram seus sistemas como insatisfatórios $($ SR_F $=20)$.

Tabela 4

Categorização da Amplitude do Sistema de Avaliação de Desempenho (Cat_Sis_Desem)

\begin{tabular}{|c|c|c|}
\hline Sigla & Descrição dos Níveis de Amplitude dos Sistemas de Avaliação de Desempenho & Quantidade \\
\hline SDF & $\begin{array}{l}\text { "Fraca": demonstra que a empresa possui um sistema de avaliação de desempenho que } \\
\text { atua com uma amplitude insatisfatória }\end{array}$ & 20 \\
\hline SDB & $\begin{array}{l}\text { "Baixa": demonstra que a empresa possui um sistema de avaliação de desempenho } \\
\text { de amplitude adequada, mas possui potencial para melhorias para atingir um bom } \\
\text { funcionamento }\end{array}$ & 2 \\
\hline SDM & $\begin{array}{c}\text { "Média": demonstra que a empresa possui um sistema de avaliação de desempenho de } \\
\text { amplitude satisfatória, apenas para uma das métricas de análise consideradas, enquanto } \\
\text { para a outra demonstra alcançar apenas uma amplitude adequada (apresenta potencial } \\
\text { para melhorias) }\end{array}$ & 8 \\
\hline SD_A & $\begin{array}{c}\text { "Alta": demonstra que a empresa possui um sistema de avaliação de desempenho de } \\
\text { uma alta amplitude, onde ambas as métricas de análise atuam com uma amplitude que } \\
\text { já apresenta maior evolução/desenvolvimento }\end{array}$ & 48 \\
\hline SD_S & $\begin{array}{c}\text { "Avançada": demonstra que a empresa possui um sistema de avaliação de desempenho } \\
\text { com amplitude avançada, pois ambas as métricas de análise apresentam o mais alto } \\
\text { índice de evolução/desenvolvimento que podem alcançar }\end{array}$ & 11 \\
\hline
\end{tabular}

Posteriormente, utilizou-se a Análise de Homogeneidade (HOMALS), modelo aplicado para estudar a relação existente entre mais de duas variáveis qualitativas. Segundo Fávero et al. (2009 p. 291), a HOMALS possibilita analisar as relações entre todas as variáveis, de forma conjunta e simultânea, a partir de uma configuração simples e bidimensional, razão pela qual seu uso se tornou aplicável às ciências sociais.

As duas técnicas têm por objetivo averiguar as associações existentes entre as variáveis consideradas em um espaço multidimensional (Figueira, 2004a,b) e são modelos de interdependência que vêm se tornando cada vez mais populares para a redução dimensional no processo de análise. Devido à facilidade de interpretação gráfica dos dados, apresentados por meio de mapeamentos percentuais, fornece uma aplicação direta mediante a retratação da correspondência de categorias de variáveis, particularmente dessas medidas em escalas nominais (Hair et al., 2009, p. 441). Esses testes estatísticos foram realizados por meio do software Stata versão 12. 


\section{Resultados e Discussão}

Por meio da ANACOR, foram avaliadas as associações Cultura Organizacional x Nível de Parceria da Controladoria (associado com a H1); Nível de Parceria da Controladoria x Amplitude dos Sistemas de Avaliação de Controle (associado com a H2); e Cultura Organizacional x Amplitude dos Sistemas de Avaliação de Desempenho (associado com a H3).

A Tabela 5 apresenta os resultados obtidos para a avaliação da associação entre Cultura Organizacional e Nível de Parceria da Controladoria.

O teste ANACOR mostrou-se estatisticamente significativo a $1 \%(\mathrm{pr}=0,0000)$, evidenciando que a associação entre cultura organizacional e níveis de parceria da controladoria não ocorreu de forma aleatória na amostra de empresas avaliadas, o que permite aceitar H1. Assim, esse achado corrobora as afirmações de Smircich (1983), para quem a cultura influencia os comportamentos dos gerentes, de tal forma que eles acabam por constituir estruturas que levam ao equilíbrio da organização. Também possibilita refletir sobre os resultados de Zheng et al. (2010) e Santos et al. (2014), que identificaram a associação da cultura organizacional com a estrutura; os primeiros, qualificando-a como mediadora em relação ao desempenho organizacional; e os demais, enfatizando sua influência sobre ele.

Tabela 5

Nível de Associação: Cultura Organizacional x Nível de Parceria da Controladoria

\begin{tabular}{cccccccc}
\hline \multirow{2}{*}{ Cat_Cult } & \multicolumn{7}{c}{ Cat_Contr } \\
\cline { 2 - 8 } & BP_F & BP_B & BP_M & BP_R & BP_A & BP_S & Total \\
\hline Cla & 1 & 0 & 1 & 7 & 12 & 9 & 30 \\
\hline Ino & 0 & 0 & 1 & 0 & 3 & 3 & 7 \\
\hline Hie & 2 & 1 & 0 & 4 & 10 & 15 & 7 \\
\hline Mer & 2 & 0 & 0 & 1 & 6 & 0 & 16 \\
\hline Cla_Hi & 0 & 1 & 0 & 0 & 0 & 1 & 3 \\
\hline Ino_Hi & 1 & 0 & 0 & 1 & 0 & 35 & 89 \\
\hline Total & 6 & 2 & 2 & 13 & 31 & & 7 \\
\hline
\end{tabular}

Nota: Pearson chi 2(25) $=62.6006 \operatorname{Pr}=0.000$

Tipificações culturais: Cla=Clã; Ino=Inovativa; Hie=Hierárquica; Mer=Mercado; Cla_Hi=Clã e Hierárquica, simultaneamente; Ino_Hi=Inovativa e Hierárquica, simultaneamente.

Nível de parceria do setor de controladoria: BP_F = Fraco; BP_B = Baixo; BP_M = Médio1; BP_R=Médio2; BP_A=Alto; BP_ $\mathrm{S}=$ Avançado.

Igualmente, para a análise da associação entre Nível de Parceria da Controladoria e Amplitude dos Sistemas de Avaliação de Controle, o teste ANACOR mostrou-se estatisticamente significativo a 1\% ( $\mathrm{pr}=0,003)$, evidenciando que a associação entre essas duas variáveis não ocorreu de forma aleatória na amostra de empresas avaliadas, o que permite aceitar $\mathrm{H} 2$. 
Os resultados apresentados na Tabela 6 permitem confirmar os achados de Franco-Santos, Lucianetti e Bourne (2012, p. 41), para quem os sistemas de avaliação de desempenho acabam por influenciar as capacidades das estruturas das organizações, considerando que a controladoria, em seus diferentes níveis, reflete esse conjunto de competências e de capacidades. Também confirmam os resultados de Zheng et al. (2010), de que a amplitude dos sistemas de avaliação de desempenho reflete diretamente as diferentes dimensões que possibilitam compreender o desempenho da organização. Confirmam, ainda, os estudos de Goretzki et al. (2013), sobre como os controllers foram transformados em business partners após a entrada de um novo CFO, o que levou à reformulação de seus papéis, e, consequentemente, modificou a atuação da controladoria, o que se refletiu nos sistemas de informação.

Tabela 6

Nível de Associação: Nível de Parceria da Controladoria x Amplitude do Sistema de Avaliação de Desempenho

\begin{tabular}{|c|c|c|c|c|c|c|}
\hline \multirow{2}{*}{ Cat_Contr } & \multicolumn{6}{|c|}{ Cat_Sis_Desem } \\
\hline & SD_F & SD_B & SD_M & SD_A & SD_S & Total \\
\hline BP_F & 5 & 1 & 0 & 0 & 0 & 6 \\
\hline BP_B & 0 & 0 & 1 & 1 & 0 & 2 \\
\hline BP_M & 1 & 0 & 1 & 0 & 0 & 2 \\
\hline BP_R & 2 & 0 & 1 & 10 & 0 & 13 \\
\hline BP_A & 6 & 1 & 3 & 19 & 2 & 31 \\
\hline BP_S & 6 & 0 & 2 & 18 & 9 & 35 \\
\hline Total & 20 & 2 & 8 & 48 & 11 & 89 \\
\hline
\end{tabular}

Nota: Pearson chi2 (20) $=41.9299 \mathrm{Pr}=0.003$

Nível de parceria do setor de controladoria: BP_F = Fraco; BP_B = Baixo; BP_M = Médio1; BP_R=Médio2; BP_A= Alto; BP_S= Avançado.

Amplitude do Sistema de Avaliação de Desempenho: SDF=Fraca; SDB=Baixa; SDM=Média; SD_A=Alta; SD_S=Avançada.

Por fim, na análise da associação entre as variáveis Cultura Organizacional e Amplitude dos Sistemas de Avaliação de Desempenho, o teste ANACOR não se mostrou estatisticamente significativo ( $p r=0,969$ ), evidenciando que a associação entre essas duas variáveis se deu de forma aleatória na amostra de empresas avaliadas, o que não permite aceitar H3. A Tabela 7 apresenta os resultados do teste.

Tabela 7

Nível de Associação: Cultura Organizacional x Amplitude do Sistema de Avaliação de Desempenho

\begin{tabular}{|c|c|c|c|c|c|c|}
\hline \multirow{2}{*}{ Cat_Cult } & \multicolumn{6}{|c|}{ Cat_Sis_Desem } \\
\hline & SD_F & SD_B & SD_M & SD_A & SD_S & Total \\
\hline $\mathrm{Cla}$ & 6 & 2 & 3 & 16 & 3 & 30 \\
\hline Ino & 1 & 0 & 1 & 5 & 0 & 7 \\
\hline Hie & 8 & 0 & 2 & 18 & 4 & 32 \\
\hline Mer & 4 & 0 & 2 & 7 & 3 & 16 \\
\hline $\mathrm{Cla}_{-} \mathrm{Hi}$ & 0 & 0 & 0 & 1 & 0 & 1 \\
\hline Ino_Hi & 1 & 0 & 0 & 1 & 1 & 3 \\
\hline Total & 20 & 2 & 8 & 48 & 11 & 89 \\
\hline
\end{tabular}

Nota. Pearson chi2 (20) $=9.9302 \mathrm{Pr}=0.969$

Tipificações culturais: $\mathrm{Cla}=\mathrm{Clã}$; Ino=Inovativa; Hie= Hierárquica; Mer=Mercado; Cla_Hi=Clã e Hierárquica, simultaneamente; Ino_Hi=Inovativa e Hierárquica, simultaneamente.

Amplitude do Sistema de Avaliação de Desempenho: SDF= Fraca; SDB=Baixa; SDM=Média; SD_A=Alta; SD_S=Avançada. 
Pode-se verificar, primeiramente, que este resultado não está em conformidade com os apresentados por Braunscheidel, Suresh e Boisnier (2010), que identificaram que a cultura organizacional pode ser considerada a base que sustenta as práticas de gestão e operações de uma empresa. Também não foi possível confirmar a posição de Bititci et al. (2012, pp. 14-15), para quem a cultura organizacional é um fator-chave para a implementação bem-sucedida de um sistema de avaliação de desempenho. Todavia, com base em nossos resultados, isso não significa dizer que a cultura organizacional não tenha associação indireta com tais sistemas. Nessa linha, pode-se considerar o posicionamento de Pothukuchi et al. (2002), para quem as áreas e unidades de negócio tendem a reagir melhor quando as práticas de gestão por elas adotadas são compatíveis com o modelo de cultura organizacional da empresa.

A fim de ampliar a compreensão dos relacionamentos entre as três variáveis estudadas, procedeuse à técnica HOMALS, avaliando a relação entre as três variáveis simultaneamente. Os resultados podem ser observados nas Tabelas 8 e 9 .

Tabela 8

Análise Conjunta 1 (HOMALS): Cultura Organizacional x Nível de Parceria da Controladoria x Amplitude do Sistema de Avaliação de Desempenho

\begin{tabular}{|c|c|c|c|c|c|}
\hline Dimension & singular value & principal inertia & chi2 & percent & cumul percent \\
\hline $\operatorname{dim} 1$ & 1 & 1 & 89.00 & 60.13 & 60.13 \\
\hline $\operatorname{dim} 2$ & .5243248 & .2749165 & 24.47 & 16.53 & 76.67 \\
\hline $\operatorname{dim} 3$ & .4449859 & .1980125 & 17.62 & 11.91 & 88.57 \\
\hline $\operatorname{dim} 4$ & .364188 & .1326329 & 11.80 & 7.98 & 96.55 \\
\hline $\operatorname{dim} 5$ & .2395453 & .0573819 & 5.11 & 3.45 & 100.00 \\
\hline Total & & 1.662944 & 148.00 & 100.00 & \\
\hline
\end{tabular}

Nota: Correspondence analysis - Number of obs = 89; Pearson chi $2(85)=148.00$;

Prob > chi2 = 0.0000; Total inertia = 1.6629; Number of dim. = 2; Exp. Inertia $(\%)=76.67$

Statistics for row and column categories in symmetric normalization.

Os resultados obtidos na HOMALS mostram a existência de associação estatisticamente significante a $1 \%(\mathrm{pr}=0,000)$ entre as três variáveis, e que tal associação não é aleatória. Os resultados da HOMALS também mostram que a associação mais intensa (medida com base na inércia, sendo as maiores destacadas na parte superior da Tabela 9), ocorre quando existem padrões de cultura organizacional claramente definidos na organização, sem a mescla de diferentes tipos de valores culturais; a maior significância ocorreu com as organizações de cultura hierárquica, seguida pelas de clã, mercado e inovativa. Tais achados estão indiretamente alinhados com os trabalhos de Gordon e DiTomaso (1992) e Lee e Yu (2004), para quem a força da cultura é um preditivo da intensidade do desempenho.

O modelo OCAI tem recebido críticas, pois é considerado um instrumento que não mensura propriamente a cultura, mas a classifica em diferentes grupos por tipos de valores culturais (Berkemeyer et al., 2015; Zoghbi-Manrique-de-Lara \& Ting-Ding, 2016). Entretanto, nesta pesquisa observa-se que, para o conjunto de empresas estudadas, aquelas que foram categorizadas claramente em um único tipo de valor cultural, com base no modelo OCAI, tiveram maior associação com níveis mais elevados de parceria na controladoria. De certa forma, pode-se considerar que a força de uma cultura, avaliada sob a ótica desse instrumento, mostra-se quando ele revela, para uma dada organização, uma tipificação cultural única, claramente identificada, diferentemente de quando o instrumento evidencia um mix de perfis culturais. 
Nas outras duas variáveis (níveis de parceria da controladoria e amplitude do sistema de avaliação de desempenho), as associações mais intensas estão presentes quando existem níveis mais elevados de parceria exercidos pelo setor de controladoria e maior amplitude dos sistemas de avaliação de desempenho (maiores inércias destacadas na parte inferior da Tabela 9).

Tabela 9

Análise Conjunta 2 (HOMALS): Cultura Organizacional x Nível de Parceria da Controladoria x Amplitude do Sistema de Avaliação de Desempenho

\begin{tabular}{|c|c|c|c|c|c|c|c|c|c|}
\hline \multirow{2}{*}{ Categories } & \multicolumn{3}{|c|}{ overall } & \multicolumn{3}{|c|}{ dimension_1 } & \multicolumn{3}{|c|}{ dimension_2 } \\
\hline & mass & quality & \%inert & coord & srcorr & contrib & coord & srcorr & Contrib \\
\hline \multicolumn{10}{|l|}{ Cultura } \\
\hline Cla & 0.337 & 0.656 & 0.098 & 0.107 & 0.024 & 0.004 & 0.762 & 0.633 & 0.374 \\
\hline Ino & 0.079 & 0.610 & 0.114 & 0.107 & 0.005 & 0.001 & -1.668 & 0.605 & 0.417 \\
\hline Hie & 0.360 & 0.435 & 0.069 & 0.107 & 0.036 & 0.004 & -0.493 & 0.399 & 0.167 \\
\hline Mer & 0.180 & 0.040 & 0.063 & 0.107 & 0.020 & 0.002 & 0.148 & 0.020 & 0.008 \\
\hline Cla_Hi & 0.011 & 1.000 & 0.595 & -9.381 & 1.000 & 0.989 & 0.000 & 0.000 & 0.000 \\
\hline Ino_Hi & 0.034 & 0.096 & 0.062 & 0.107 & 0.004 & 0.000 & 0.736 & 0.093 & 0.035 \\
\hline \multicolumn{10}{|l|}{ BP_Sist_Inf } \\
\hline BP F/SDF & 0.056 & 0.008 & 0.051 & 0.107 & 0.007 & 0.001 & 0.018 & 0.000 & 0.000 \\
\hline BP F/SDB & 0.011 & 0.570 & 0.013 & 0.107 & 0.006 & 0.000 & 1.454 & 0.564 & 0.045 \\
\hline BP B/SDM & 0.011 & 0.267 & 0.012 & 0.107 & 0.006 & 0.000 & -0.941 & 0.260 & 0.019 \\
\hline $\mathrm{BP} B / \mathrm{SD} A$ & 0.011 & 1.000 & 0.595 & -9.381 & 1.000 & 0.989 & 0.000 & 0.000 & 0.000 \\
\hline $\mathrm{BP} \mathrm{M} / \mathrm{SDF}$ & 0.011 & 0.570 & 0.013 & 0.107 & 0.006 & 0.000 & 1.454 & 0.564 & 0.045 \\
\hline $\mathrm{BP} \mathrm{M} / \mathrm{SDM}$ & 0.011 & 0.454 & 0.079 & 0.107 & 0.001 & 0.000 & -3.180 & 0.453 & 0.217 \\
\hline BP R/SDF & 0.022 & 0.105 & 0.006 & 0.107 & 0.026 & 0.000 & 0.257 & 0.079 & 0.003 \\
\hline BP R/SDM & 0.011 & 0.570 & 0.013 & 0.107 & 0.006 & 0.000 & 1.454 & 0.564 & 0.045 \\
\hline $\mathrm{BP} R / \mathrm{SD} A$ & 0.112 & 0.606 & 0.023 & 0.107 & 0.033 & 0.001 & 0.613 & 0.573 & 0.081 \\
\hline BP A/SDF & 0.067 & 0.968 & 0.012 & 0.107 & 0,040 & 0.001 & -0.711 & 0.928 & 0.065 \\
\hline $\mathrm{BP} A / \mathrm{SDB}$ & 0.011 & 0.570 & 0.013 & 0.107 & 0.006 & 0.000 & 1.454 & 0.564 & 0.045 \\
\hline BP A/SDM & 0.034 & 0.138 & 0.037 & 0.107 & 0.006 & 0.000 & 0.673 & 0.132 & 0.029 \\
\hline $\mathrm{BP} A / S D \mathrm{~A}$ & 0.213 & 0.169 & 0.018 & 0.107 & 0,082 & 0.002 & 0.151 & 0.087 & 0.009 \\
\hline BP A/SD S & 0.022 & 0.267 & 0.024 & 0.107 & 0.006 & 0.000 & -0.941 & 0.260 & 0.038 \\
\hline BP S/SDF & 0.067 & 0.598 & 0.008 & 0.107 & 0.055 & 0.001 & 0.461 & 0.542 & 0.027 \\
\hline BP S/SDM & 0.022 & 0.105 & 0.006 & 0.107 & 0.026 & 0.000 & 0.257 & 0.079 & 0.003 \\
\hline BP S/SD A & 0.202 & 0.955 & 0.049 & 0.107 & 0.028 & 0.002 & -0.844 & 0.927 & 0.275 \\
\hline BP S/SD S & 0.101 & 0.347 & 0.027 & 0.107 & 0.025 & 0.001 & 0.526 & 0.321 & 0.053 \\
\hline $\begin{array}{l}\text { Vota: Nível de } \\
\text { BP_S=Avançado } \\
\text { Tipificações cul } \\
\text { no_Hi=Inovativ } \\
\text { Amplitude do S }\end{array}$ & rceria do & tor de cor & oladoria: & $\begin{array}{l}\text { _F = Fraco } \\
\text { erárquica }\end{array}$ & $P_{-} B=B a$ & $\begin{array}{l}; \mathrm{BP}_{-} \mathrm{M}= \\
; \mathrm{Cla}+\mathrm{Hi}=\end{array}$ & $\begin{array}{l}\text { ćdio1; BP } \\
\text { a e Hierár }\end{array}$ & $\begin{array}{l}\text { Médio2; } \\
\text { ca simult }\end{array}$ & A=Alto; \\
\hline
\end{tabular}


Esses resultados sugerem que quando o controller está mais próximo dos gestores, entendendo os fatores que influenciam o resultado das áreas por eles comandadas, ele consegue influenciar o aumento da amplitude do sistema de avaliação de desempenho, podendo mensurar os resultados de forma mais ampla (Frezatti et al., 2010) e, ao mesmo tempo, aprofundada, podendo até chegar ao nível da transação, conforme defendido pelo modelo GECON (Catelli, 2001). Em síntese, na linguagem de mercado, tais ações levam ao que se convencionou chamar "obtenção de diferentes granularidades da informação". Além disso, por estar próximo, ele consegue melhorar a amplitude dos sistemas de informação ao auxiliar os gestores nos processos de planejamento, controle e tomada de decisão, contribuindo para o seu desempenho e o da controladoria (Oyadomari et al., 2014).

Em síntese, de acordo com o resultado desses testes, pode-se inferir que tipificações mais fortes de cultura organizacional, ou em outras palavras, definidas claramente, estão associadas a maiores níveis de parceria da controladoria, e que essas estão associadas à maior amplitude de sistemas de avaliação de desempenho, apesar de não ter sido encontrada uma associação direta entre modelo de cultura organizacional e amplitude dos sistemas de avaliação de desempenho. Nesse sentido, os resultados, embora não de forma semelhante, divergem dos resultados de Henri (2006), para quem, independentemente do tipo de cultura (flexível ou de controle), existe associação positiva com a diversidade das medidas de desempenho, que é um dos componentes da amplitude do sistema de avaliação de desempenho.

Entretanto, não se pode negar que o modelo de cultura organizacional influencia a amplitude dos sistemas de avaliação de desempenho, o que pode ocorrer de forma indireta, considerando que tais tipos de cultura influenciam as áreas de controladoria, e essas operacionalizam modelos mais ou menos amplos. Nesse sentido, a controladoria seria um "mediador" do relacionamento entre a cultura organizacional e os sistemas de avaliação de desempenho, o que confirmaria as afirmações de Zheng et al. (2010), que identificaram que a estrutura é mediadora do efeito da cultura organizacional no desempenho (ficácia empresarial).

Os achados desta pesquisa também comprovam empiricamente os de Ho et al. (2014), para quem empresas alinhadas em torno de consenso - possível, entre outras razões, como decorrência de uma clara cultura organizacional (Cameron \& Quinn, 2005) -, têm melhores condições para o desenvolvimento de tecnologias de gestão, papel central da área de controladoria. Isto contribui para alcançar a congruência de objetivos organizacionais e para o desenvolvimento e disseminação de práticas de gestão, tais como os sistemas de avaliação de desempenho (Neely et al., 2000).

\section{Conclusões}

Pode-se considerar que esta pesquisa atendeu ao objetivo principal de compreender as associações existentes entre as diferentes intensidades de tipificações culturais organizacionais, o nível de parceria exercido pelo setor de controladoria e a amplitude dos sistemas de avaliação de desempenho organizacional.

Os dados obtidos foram analisados por meio de análise multivariada (ANACOR, HOMALS), o que possibilitou identificar, na amostra estudada, a existência de associação estatisticamente significante entre Modelo de Cultura Organizacional e Níveis de Parceria da Controladoria e, ainda, entre Níveis de Parceria da Controladoria e Amplitude do Sistema de Avaliação de Desempenho. Porém, não foi observada a existência de associação estatisticamente significante entre Modelo de Cultura Organizacional e Amplitude do Sistema de Avaliação de Desempenho.

Tais achados permitem inferir que a controladoria, ao materializar as práticas de gestão na organização (papel fundamental de sua atuação), pode ser mais assertiva, se houver mais intensidade de um determinado tipo de cultura organizacional. Assim, sob esse enfoque, pode-se caracterizar a Controladoria como mediadora entre a Cultura Organizacional e os Sistemas de Avaliação de Desempenho. 
Os resultados também revelam a importância de uma atuação mais participativa da área de controladoria, como meio de ampliar o sistema de avaliação de desempenho, o que constitui outro fator, além da abordagem da Teoria da Contingência, que explica a amplitude dos sistemas de avaliação de desempenho.

Em termos de práticas organizacionais, nossos resultados sugerem que a controladoria, com base em interações com as áreas usuárias de seus serviços, interpreta os elementos da cultura organizacional e influencia o escopo do sistema de avaliação de desempenho. Se a cultura é mais do tipo participativa e democrática, os serviços da controladoria, em termos normativos, deveriam abranger diferentes usuários das informações, em diferentes áreas e níveis organizacionais, fornecendo tais informações para validar, ou não, as diferentes interpretações desses usuários quanto à formulação do diagnóstico e das propostas de solução de problemas, além de acompanhar essas iniciativas. Com isso, o escopo do sistema de avaliação de desempenho deveria ser do tipo mais coletivo, baseado em metas interdependentes e que priorizasse o desempenho do grupo. Por outro lado, se a cultura é do tipo hierárquica, à luz da Teoria da Contingência, o papel da controladoria na interação com os usuários deveria priorizar informações para os tomadores de decisão, resultando em um sistema de avaliação de desempenho mais focado nos níveis hierárquicos que tomam as decisões.

Com base nos resultados, pode-se recomendar que a alta administração desenvolva ações para “impregnar' a cultura nas pessoas, de forma que ela esteja presente nas ações rotineiras da organização, pois isso facilita a atuação da controladoria como uma parceira de negócios, apesar de as técnicas estatísticas não permitirem avaliar a causalidade entre as variáveis estudadas. Tais resultados oferecem uma visão complementar àquela de Dambrin, Lambert \& Sponem (2007) e Goretzki et al. (2013), em que os autores inferem que a mudança cultural ocorre por meio da ação de novos controllers, que passam a adotar posturas colaborativas, ampliando a participação das controladorias em que eles atuam, e, consequentemente, influenciando a cultura das respectivas organizações.

Entende-se, também, que os resultados da pesquisa contribuem para o desenvolvimento de conhecimento acadêmico, na medida em que fornece elementos empíricos para refletir sobre a influência da cultura organizacional nas práticas de gestão, por meio do desenvolvimento e uso de constructos e de instrumentos de investigação, possibilitando uma reflexão além dos conceitos da Teoria da Contingência, conforme já mencionado.

Por outro lado, os resultados não permitem assegurar quais os tipos de cultura organizacional que estão associados a níveis específicos de parceria na controladoria e de amplitude nos sistemas de avaliação de desempenho. Entretanto, eles mostram que a clareza na definição da cultura organizacional, seja ela de qualquer tipo, produz efeitos positivos na controladoria, levando-a a práticas atuantes e participativas. Por fim, a análise estatística utilizada não permite afirmar que existe uma relação causal entre as variáveis estudadas, nem mesmo fazer generalizações; assim, futuros estudos que verifiquem esta causalidade serão úteis para ampliar o conhecimento sobre o tema.

\section{Referências}

Arenales, A. D. R. (2016). O papel da controladoria como business partner (Dissertação de Mestrado). Universidade Presbiteriana Mackenzie, São Paulo. http://tede.mackenzie.br/jspui/bitstream/ tede/2794/2/ALEXANDRE\%20DANIEL\%20ROCA\%20ARENALES.pdf.

Barreto, L. M. T. S., Kishore, A., Reis, G. G., Baptista, L. L., \& Medeiros, C. A. F. (2013). Cultura organizacional e liderança: uma relação possível? Revista de Administração, 48(1), 34-52. https:// doi.org/10.5700/rausp1072

Berkemeyer, N., Junker, R., Bos, W., \& Müthing, K. (2015). Organizational cultures in education: Theorybased use of an instrument for identifying school culture. Journal for Educational Research Online, 7(3), 86-102. http://www.j-e-r-o.com 
Beuren, I. M., Fachini, G. J., \& Nascimento, S. (2010). Evidências de isomorfismo nas funções da controladoria das empresas familiares têxteis de Santa Catarina. Revista Contemporânea de Contabilidade, 7(13), 35-62. https://doi.org/10.5007/2175-8069.2010v7n13p35

Bititci, U., Mendibil, K., Nudurupati, S., Turner, T., \& Garengo, P. (2006). Dynamics of performance measurement and organizational culture. International Journal of Operations and Production Management, 26(12), 1325-1350. https://doi.org/10.1108/01443570610710579.

Bititci, U., Garengo, P., Dörfler, V., \& Nudurupati, S. (2012). Performance measurement: Challenges for tomorrow. International Journal of Management Reviews, 14(3), 305-327. https://doi.org/10.1111/ j.1468-2370.2011.00318.x

Bonisenha, C. N., \& d'Angelo, M. J. (2018). O papel da cultura organizacional no desempenho operacional de uma instituição de ensino superior à luz dos indicadores de qualidade SINAES. Revista Base (Administração e Contabilidade) da UNISINOS, 15(4). http://www.revistas.unisinos.br/index.php/ base/article/view/base.2018.154.05

Borinelli, M. L. (2006). Estrutura conceitual básica de controladoria: Sistematização à luz da teoria e da práxis (Tese de Doutorado). Faculdade de Economia, Administração e Contabilidade, Universidade de São Paulo, São Paulo. https://doi.org/10.11606/T.12.2006.tde-19032007-151637

Braunscheidel, M. J., Suresh, N. C., \& Boisnier, A. D. (2010). Investigating the impact of organizational culture on supply chain integration. Human Resource Management, 49(5), 883-911. https://doi. org/10.1002/hrm.20381

Cagno, E., Neri, A., Howard, M., Brenna, G., \& Trianni, A. (2019). Industrial sustainability performance measurement systems: A novel framework. Journal of Cleaner Production, 230, 1354-1375. https:// doi.org/10.1016/j.jclepro.2019.05.021

Cameron, K. S., \& Quinn, R. E. (2005). Diagnosing and changing organizational culture. Based on the Competing Values Framework. Jossey-Bass.

Cao, Z., Huo, B., Li, Y., \& Zhao, X. (2015). The impact of organizational culture on supply chain integration: A contingency and configuration approach. Supply Chain Management, 20(1), 24-41. https://doi. org/10.1108/SCM-11-2013-0426

Carmona, L. J. M., Silva, T. B. J, \& Gomes, G. (2018). Cultura organizacional, inovação e desempenho em escritórios de contabilidade brasileiros. Contabilidade Vista \& Revista, 29(3), 121-145. https://doi. org/10.22561/cvr.v29i3.3936

Catelli, A. (2001). Controladoria: Uma abordagem da gestão econômica (2a ed.). Atlas.

Dambrin, C., Lambert, C., \& Sponem, S. (2007). Control and change - Analyzing the process of institutionalization. Management Accounting Research, 18(2), 172-208. https://doi.org/10.1016/j. mar.2007.02.003

Dillman, D. A. (2000). Mail and internet surveys: The tailored design method. John Wiley \& Sons.

Dubey, R., Gunasekaran, A., Childe, S. J., Roubaud, D., Wamba, S. F., Giannakis, M., \& Foropon, C. (2019). Big data analytics and organizational culture as complements to swift trust and collaborative performance in the humanitarian supply chain. International Journal of Production Economics, 210, 120-136. https://doi.org/10.1016/j.ijpe.2019.01.023

Fávero, L. P. L., Belfiore, P. P., Silva, F. L., \& Chan, B. L. (2009). Análise de dados: Modelagem multivariada para tomada de decisões. Elsevier.

Figueira, A. P. C. (2004a). Procedimento HOMALS - Instrumentalidade em estudos nas áreas das Ciências Sociais e Humanas: Um exemplo ilustrativo. Psychologica - Revista da Faculdade de Psicologia e de Ciências da Educação da Universidade de Coimbra, 40/41, 601-610. https:// doi.org/10.14195/1647-8606 
Figueira, A. P. C. (2004b). Faça você mesmo. Procedimento ANACOR passo a passo. Revista IberoAmericana de Educación. https://rieoei.org/historico/deloslectores/795Couceiro.PDF

Franco, M., \& Bourne M. (2003). Factors that play a role in "managing through measures". Management Decision, 41(8), 698-710. https://doi.org/10.1108/00251740310496215

Franco-Santos, M., Lucianetti, L., \& Bourne, M. (2012). Contemporary performance measurement systems: A review of their consequences and a framework for research. Management Accounting Research, 23(2), 79-19. https://doi.org/10.1016/j.mar.2012.04.001

Frezatti, F., Relvas, T. R. S., \& Junqueira, E. (2010). BSC e a estrutura de atributos da contabilidade gerencial: Uma análise no ambiente brasileiro. Revista de Administração de Empresas, 50(2), 187-198. https:// doi.org/10.1590/S0034-75902010000200005

Garengo, P., Biazzo, S., \& Bititci, U. S. (2005). Performance measurement systems in SMEs: A review for a research agenda. International Journal of Management Reviews, 7(1), 25-47. https://doi.org/10.1111/ j.1468-2370.2005.00105.x

Globerson, S. (1985). Issues in developing a performance criteria system for an organization. International Journal of Production Research, 23(4), 639-646. https://doi.org/10.1080/00207548508904734

Goretzki, L., Strauss, E., \& Weber, J. (2013). An institutional perspective on the changes in management accountants' professional role. Management Accounting Research, 24(1), 41-63. https://doi. org/10.1016/j.mar.2012.11.002

Gordon, G. G., \& DiTomaso, N. (1992). Predicting corporate performance from organizational culture. Journal of Management Studies, 29(6), 783-798. https://doi.org/10.1111/j.1467-6486.1992.tb00689.x

Hair, J. F., Black, W. C., Babin, B. J., Anderson, R. E., \& Tatham, R. L. (2009). Análise multivariada de dados. Bookman.

Hansen, G. S., \& Wernerfelt, B. (1989). Determinants of firm performance: The relative importance of economic and organizational factors. Strategic Management Journal, 10(5), 399-411. https://doi. org/10.1002/smj.4250100502

Hartnell, C. A., Ou, A. Y., \& Kinicki, A. (2011). Organizational culture and organizational effectiveness: A meta-analytic investigation of the competing values framework's theoretical suppositions. Journal of Applied Psychology, 96(4), 677-694 https://doi.org/10.1037/a0021987.supp

Henri, J. F. (2006). Organizational culture and performance measurement systems. Accounting, Organizations and Society, 31(1), 77-103. https://doi.org/10.1016/j.aos.2004.10.003

Ho, J. L. Y., Wu, A., \& Wu, S. Y. C. (2014). Performance measures, consensus on strategy implementation, and performance: Evidence from the operational level of organizations. Accounting, Organizations, and Society, 39(1), 38-58. https://doi.org/10.1016/j.aos.2013.11.003

Hock-Doepgen, M., Clauss, T., Kraus, S., \& Cheng, C. F. (2021). Knowledge management capabilities and organizational risk-taking for business model innovation in SMEs. Journal of Business Research, 130, 683-697. https://doi.org/10.1016/j.jbusres.2019.12.001

Hora, H. R. M., Monteiro, G. T. R., \& Arica, J. (2010). Confiabilidade em questionários para qualidade: Um estudo com o coeficiente Alfa de Cronbach. Produto \& Produção, 11(2), 85-103. https://doi. org/10.22456/1983-8026.9321

Järvenpää, M. (2007). Making business partners: A case study on how management accounting culture was changed. European Accounting Review, 16(1), 99-142. https://doi.org/10.1080/09638180701265903

Kotrba, L. M., Gillespie, M. A., Schmidt, A. M., Smerek, R. E., Ritchie, S. A., \& Denison, D. R. (2012). Do consistent corporate cultures have better business performance? Exploring the interaction effects. Human Relations, 65(2), 241-262. https://doi.org/10.1177/0018726711426352 
Lee, S. K. J., \& Yu, K. (2004). Corporate culture and organizational performance. Journal of Managerial Psychology, 19(4), 340-359. https://doi.org/10.1108/02683940410537927

Lunkes, R. J., Schnorrenberger, D., \& Alexandre, K. (2016). Funções da controladoria: Um estudo sobre a percepção dos gestores e do controller em uma empresa de tecnologia. Revista ESPACIOS, 37 (3). http://www.revistaespacios.com/a16v37n03/16370306.html

Maskell, B. H. (1991). Performance measurement for world class manufacturing. A model for American companies. Productivity Press.

Melo, I. I. S. L., \& Paulo, E. (2000). Controladoria como órgão administrativo e ramo do conhecimento. Anais do IX Congresso Brasileiro de Custos - ABC. https://anaiscbc.emnuvens.com.br/anais/article/view/2688

Merchant, K. A., \& Van der Stede, W. A. (2007). Management control systems: Performance measurement, evaluation and incentives. Pearson Education. https://doi.org/10.1108/18325910810878982

Miranda, C. S., Riccio, E. L., \& Miranda, R. A. M. (2012). Atividades da contabilidade gerencial sob a ótica de docentes e profissionais de mercado. Revista Ambiente Contábil, 5(1), 112-131. https://ojs.ccsa. ufrn.br/index.php/contabil/article/view/331

Naranjo-Valencia, J. C., Jiménez-Jiménez, D., \& Sanz-Valle, R. (2011). Innovation or imitation? The role of organizational culture. Management Decision, 49(1), 55-72 https://doi. org/10.1108/00251741111094437

Neely, A., Bourne, M., \& Kennerly, M. (2000). Performance measurement system design: Developing and testing a process-based approach. International Journal of Operations \& Production Management, 20(10), 1119-1145. https://doi.org/10.1108/01443570010343708

Neely, A., Gregory, M., \& Platts, K. (1995). Performance measurement system design: A literature review and research agenda. International Journal of Operations \& Production Management, 15(4), 80-117. https://www.leanway.com.br/wp-content/uploads/Neely.pdf

O’Reilly, C. A., III, Caldwell, D. F., Chatman, J. A., \& Doerr, B. (2014). The promise and problems of organizational culture: CEO personality, culture, and firm performance. Group \& Organization Management, 39(6), 595-625. https://doi.org/10.1177/1059601114550713

Oyadomari, J. C. T., Aguiar, A. B., Yen-Tsang, C., Cardoso, R. L., \& Lima, R. G. D. (2014). Associações entre informações, desempenho da controladoria, desempenho gerencial e organizacional: Um estudo exploratório. Revista de Educação e Pesquisa em Contabilidade, 8(3), 309-325. https://doi. org/10.17524/repec.v8i3.1087

Parente, P. H. N., De Luca, M. M. M., Lima, G. A. S. F., \& Vasconcelos, A. C. (2018). Cultura organizacional e desempenho nas empresas estrangeiras listadas na NYSE. Revista de Contabilidade e Organizações, 12, 139-161. https://doi.org/10.11606/issn.1982-6486.rco.2018.139161

Pothukuchi, V., Damanpour, F., Choi, J., Chen, C. C., \& HoPark, S. (2002). National and organizational culture differences and international joint venture performance. Journal of International Business Studies, 33(2), 243-265. https://doi.org/10.1057/palgrave.jibs.8491015

Rieg, R. (2018). Tasks, interaction and role perception of management accountants: Evidence from Germany. Journal of Management Control, 29(2), 183-220. https://doi.org/10.1007/s00187-018-0266-0

Santos, N. M. B. F. (1998). Cultura e desempenho organizacional: Um estudo empírico em empresas brasileiras do setor têxtil. Revista de Administração Contemporânea, 2(1), 47-76. http://dx.doi. org/10.1590/S1415-65551998000100004

Santos, N., Bronzo, M., Oliveira, M. P. V., \& Resende, P. T. V. (2014). Cultura organizacional, estrutura organizacional e gestão de pessoas como bases para uma gestão orientada por processos e seus impactos no desempenho organizacional. BBR-Brazilian Business Review, 11(3), 106-129. https:// www.redalyc.org/articulo.oa?id=123031118005 
Schein, E. H. (1984). Coming to a new awareness of organizational culture. Sloan Management Review, 25(2), 3-16. http://mbs-thread.s3.amazonaws.com/pdf_attachments/documents/14727/original/ Pre-reading_-_Schein_-_Coming_to_a_new_awareness_of_organisational_culture_-_1984.pdf

Schein, E. H. (2010). Organizational culture and leadership (Vol. 2). John Wiley \& Sons.

Silva, M. B. D. (2017). A contribuição do controller como business partner: Sistematização de atividades sob a ótica do sistema de gestão do desempenho (Dissertação de Mestrado). Universidade Presbiteriana Mackenzie, São Paulo. http://tede.mackenzie.br/jspui/handle/tede/3189

Silva, S., Nuzum, A. K., \& Schaltegger, S. (2019). Stakeholder expectations on sustainability performance measurement and assessment. A systematic literature review. Journal of Cleaner Production, 217, 204-215. https://doi.org/10.1016/j.jclepro.2019.01.203

Smircich, L. (1983). Concepts of culture and organizational analysis. Administrative Science Quarterly, 28(3), 339-358. https://doi.org/10.2307/2392246

Sony, M., Antony, J., \& Douglas, J. A. (2020). Essential ingredients for the implementation of Quality 4.0: A narrative review of literature and future directions for research. The TQM Journal, 32(4), 779793. https://doi.org/10.1108/tqm-12-2019-0275

Tarifa, M. R., Crozatti, J., Espejo, M. M. S. B., \& Almeida, L. B. (2011). A cultura organizacional influencia as práticas de controladoria? Um estudo de campo baseado no competing value model. Revista Ciências Administrativas, 17(2), 323-350. https://www.redalyc.org/articulo.oa?id=475647553001

Tarifa, M. R., \& Almeida, L. B. (2018). Cultura organizacional e práticas de contabilidade gerencial no agronegócio cooperativo. Revista Universo Contábil, 14(1), 93-114. https://doi.org/10.4270/RUC.2018105

Tian, M., Deng, P., Zhang, Y., \& Salmador, M. P. (2018). How does culture influence innovation? A systematic literature review. Management Decision, 56(5), 1088-1107. https://doi.org/10.1108/md-05-2017-0462

Weber, J. (2011). The development of controller tasks: Explaining the nature of controllership and its changes. Journal of Management Control, 22(1), 25-46. https://doi.org/10.1007/s00187-011-0123-x

Zheng, W., Yang, B., \& McLean, G. N. (2010). Linking organizational culture, structure, strategy, and organizational effectiveness: Mediating role of knowledge management. Journal of Business Research, 63(7), 763-771. https://doi.org/10.1016/j.jbusres.2009.06.005

Zoghbi-Manrique-de-Lara, P., \& Ting-Ding, J. M. (2016). The influence of corporate culture and workplace relationship quality on the outsourcing success in hotel firms. International Journal of Hospitality Management, 56, 66-77. https://doi.org/10.1016/j.ijhm.2016.04.012

\section{Apêndice 1 - Instrumento de Pesquisa}

\section{Parte 3 O setor de Controladoria:}

1. Gera relatórios que reportam a situação econômica da empresa.

2. Entrega relatórios de suporte apenas ao departamento ao qual se reporta.

3. Gera relatórios de suporte para os demais setores organizacionais.

4. Presta informações aos demais setores organizacionais, que são utilizadas no processo decisório destes departamentos, e para o estabelecimento de suas estratégias.

5. Entrega os relatórios por ele configurados, e presta suporte para garantir a sua compreensão.

6. Busca apurar com os demais setores organizacionais informações relevantes para os seus processos decisórios - a fim de garantir que tais informações lhe sejam fornecidas. 
7. Apresenta, seja à alta administração ou aos demais departamentos organizacionais, informações (mesmo que não solicitadas) que considera relevantes para o processo decisório organizacional.

8. Quando necessário, gera informações para finalidades específicas (sob demanda).

9. Age de forma independente e crítica, livre para expressar sua opinião à alta administração acerca dos resultados por ele apurados (apresentando assim o seu parecer).

10. Desenvolve modelos de propostas e projetos a serem implementados na companhia - a fim de que estes contribuam para o desenvolvimento e continuidade organizacional.

11. Gera dados que são utilizados para o planejamento organizacional (ou seja, para o estabelecimento de metas e estratégias).

12. Gera dados que são utilizados para avaliar se as metas e estratégias organizacionais estão sendo alcançadas conforme planejado.

13. Envolve-se e influencia o desenvolvimento das metas e estratégias dos demais setores - a fim de garantir que as metas definidas para eles estejam alinhadas com os objetivos traçados para a companhia.

14. É responsável por coordenar e alinhar as atividades desenvolvidas na companhia - a fim de garantir que os objetivos e metas organizacionais sejam alcançados.

\section{Parte 4 Avaliação de Desempenho = AD}

1. Existe um sistema formal de $\mathrm{AD}$ na organização em que atuo.

2. O objetivo de cada medida utilizada para se realizar uma $\mathrm{AD}$ na companhia é esclarecido (sendo exposto de forma clara e precisa).

3. As medidas adotadas para a AD na companhia são definidas com base nas estratégias da empresa e relacionadas às atividades avaliadas.

4. Ao coletar dados para a AD da organização, o método utilizado é sempre claro e objetivo.

5. Há um método/cálculo claro e objetivo utilizado para a $\mathrm{AD}$ da organização.

6. A opinião de todos na empresa (clientes, funcionários e gerentes) é considerada ao se realizar o processo de montagem/estabelecimento das medidas de $\mathrm{AD}$ da companhia.

7. As medidas adotadas para a AD da companhia se baseiam nas características e particularidades da empresa.

8. As medidas e métodos utilizados para a $\mathrm{AD}$ da companhia são flexíveis - abertos a ajustes e adequações, quando necessário.

9. As medidas adotadas para a $\mathrm{AD}$ da companhia permitem o processo de avaliação da empresa frente à sua concorrência - facilitando a incorporação de melhores práticas percebidas e/ou o aperfeiçoamento de seus próprios métodos.

10. As medidas adotadas para a $\mathrm{AD}$ da companhia baseiam-se exclusivamente nos dados apresentados em suas medidas financeiras e contábeis - não levando em consideração quaisquer outras informações ou dados disponíveis.

11. Os critérios adotados para a $\mathrm{AD}$ de um setor são definidos pela própria unidade organizacional.

12. Os critérios adotados para a $\mathrm{AD}$ da companhia são bem definidos e seus objetivos de avaliação são claros.As medidas adotadas para a $\mathrm{AD}$ da companhia levam em consideração as medidas financeiras e "não financeiras" (aumento do número de clientes, aumento da participação de mercado, etc.) geradas ao final do período analisado.

13. As medidas adotadas para a $\mathrm{AD}$ da companhia são simples e fáceis de utilizar/aplicar.

14. As medidas adotadas para a $\mathrm{AD}$ da companhia desencadeiam ações rápidas das partes envolvidas.

15. As medidas adotadas para a AD estimulam a melhoria contínua das partes envolvidas - em vez de apenas monitorá-las. 